\title{
Four-Factor based Privacy Preserving Biometric Authentication and Authorization Scheme for Enhancing Grid Security
}

\author{
G. Jaspher Willsie Kathrine \\ Department of Information Technology \\ Karunya University, Tamil Nadu, India
}

\author{
E. Kirubakaran \\ Director (Outsourcing), BHEL \\ Trichy, Tamil Nadu, India
}

\begin{abstract}
Increase in the data sharing of distributed data has resulted in resource usage being more and more distributed. The distributed nature of data and its need to be accessed has resulted in the grid environment. Authentication is considered as the first step of security requirement for any grid environment against potential threats. This paper proposes an authentication method which is based not only on the password and the user ID but also on the biometric input and the position of the user. By enhancing the security at the entry point of Virtual Organization (VO), the grid environment is secured in its first step. The same biometric and position data used for authentication can further be used for authorization purposes so as to reduce the cost and time of storing different data for different purposes. A FourFactor based Privacy Preserving Biometric (4F2PB) authentication scheme for a grid environment is proposed which can work on the existing Network Framework. The proposed authentication scheme optimises the security required for the entry level user and prevents malicious user from entering into the grid environment.
\end{abstract}

\section{Keywords:}

Grid Computing, Authentication, Virtual Organisation, Biometric Data.

\section{INTRODUCTION}

Grid computing involves sharing heterogeneous resources which are located in geographically distributed places belonging to different administrative domains [1]. Grid data sharing is not file exchange but rather access to computers, software, data and other resources. Grid involves the creation of a dynamic Virtual Organization (VO). Each virtual organization comprises of users and their resources and any other services (S) joined by a common goal [2]. Each of the user or resource is available from different administrative domains (DO). Each user or resource has its own trust policy which requires a local to global and global to local mapping of the access policies as discussed in [3].

The basic security for the Globus Toolkit (GT 4) is the Grid Security Infrastructure (GSI) in C and Java [4], [5]. It depends on the Public Key Infrastructure (PKI), X.509 Proxy certificates and TLS for authentication. GSI involves third-party verification for the purpose of authorization. The GSI security is secure enough but has scalability problems [5]. All of the existing security schemes are based on the user name and the password which belongs to a two factor authentication scheme. The proposed authentication scheme optimizes the security of a grid environment by adding more features like biometric data and the position of the user during and after authentication.

\section{RELATED WORK}

User authentication has been in discussion for a long time to enhance the security of any system at the entry level itself. Methods such as password based systems and ID based systems have been used. A hash-chain based remote user authentication in which all the passwords are encoded is given in [6]. In all the initial remote based authentication systems, a verifier table is to be placed in the server side which becomes a problem if the server is compromised. In order to avoid maintaining a verifier table Hwang et al., proposed a non-interactive smart card based scheme without verifier tables [7]. A finger print based remote user authentication scheme was proposed in [8]. This scheme was found to be vulnerable to masquerade attacks and many other attacks [9], [10]. In [11], [12], [13], the biometric data itself is taken as a key for encryption/decryption. The secret data is extracted by using the biometric template as the key. The biometric data is to be stored in the server side and used for comparison. But for effective Biometric authentication, the process is to be done in the client side [14] to avoid any problem due to the server being compromised [15]. In [16], the method has been optimized with the matching being done in the server side. But the server does not store any biometric data in its database thereby protecting the privacy of the user.

The method in [16] provides a three factor authentication which is password - something the user knows; smart card something the user has; biometrics - something the user is. A further enhancement to this type of authentication is to add a fourth factor thereby providing a four factor authentication [17]. The fourth factor can be the addition of location of the user - someplace the user is. This fourth factor can be implemented by using the data obtained from the cookies of a user's web browser or computer or from the Global Positioning System (GPS) or the IP address location process. The fourth factor addition enhances the security criteria required for a vast distributed system such as a military or medical or research or Banking Grid environments. The military data sharing requirements take into consideration the place in which the user is positioned so as to find the location of any valid/invalid user. So, the sensitive areas of application require security with some amount of privacy preservation. By combining the biometric data with passwords and the location of the user, the security factors are further enhanced. The next section shows the methodology of the proposed Four-Factor based Privacy Preserving Biometric (4F2PB) authentication system. 


\section{SECURITY FRAMEWORK FOR A GRID SYSTEM}

The existing solution uses Open Grid Services Architecture (OGSA) architecture [18]. The OGSA architecture uses the WS-Security services for authentication and authorization. The existing system based on OGSA and GSI have some basic security solutions for solving the authentication and authorization criteria. The scalability, heterogeneity and increase in attacks have led to the need of a new security framework which is based on the existing architecture with additional features to tackle the day to day attacks. The Control Architecture for Computational Grids is shown in Figure 1. The main subcomponents of this proposed system in Figure 1 are,

- A Security Client (SC)

- A Security Manager (SM)

- A Chief Security Manager (CSM)

The components are:
Service (S) - Service/resource is the resource provider of the grid environment. Each resource provider can be a private individual or a member of another organization. Service Policy (SP) - Every service holder has their own Service Policy based on the VO and the domain to which they belong. Domain (DO) - The Organization to which each individual service resides in is a Domain. It is not necessary that all the members of the same domain belong to the same VO. Virtual Organization (VO) - A VO temporarily aggregates resources of different domains to achieve a common goal. Virtual Organization Policy (VP) - This is the policy set up of the VO which is followed by all the members of the VO. This policy is set based on a combination of the DO, SP, Authentication Policy (AuP), Authorization Policy (AuZP) and any Other Policy (OP). The policy setting determines the strength of the security. The Security Client (SC) which is a security feature present within each service/resource of the Virtual Organization (VO).

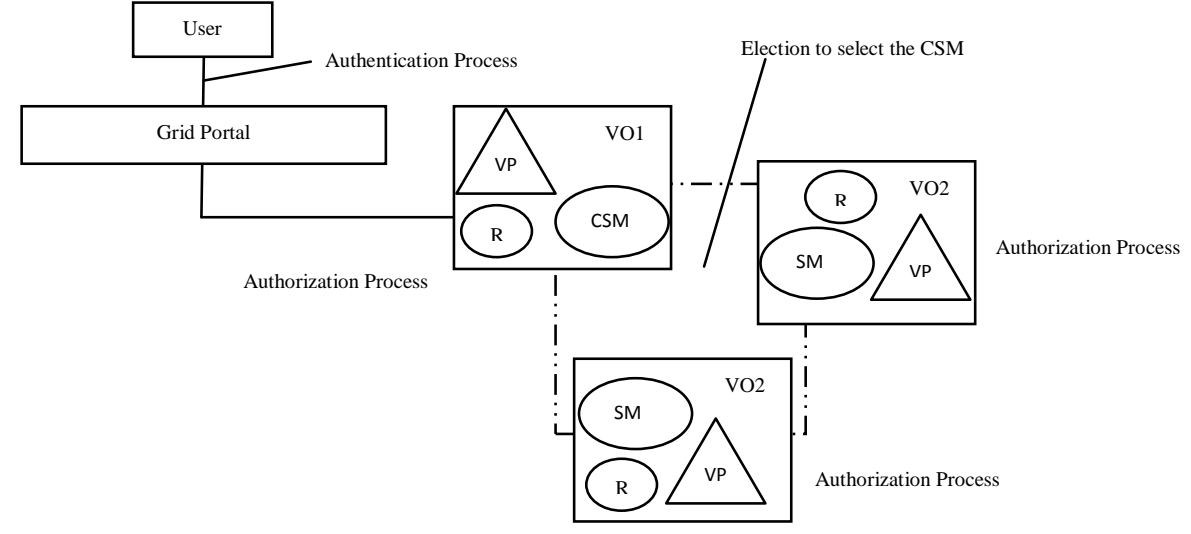

Figure 1. Control Architecture for Computational Grid

This security feature, will analyze/study the user requests and the processes for any impending security compromise. Once a compromise is identified, the detail is immediately sent to the VO's Security Manager (SM). The attack if any is then sent to the Chief Security Manager (CSM) which further analyses the input based on the audit log. If any attack is identified by the CSM, it is informed to the various SM's of the other VO's to avoid the attack spreading further in the Grid environment.

\section{FOUR-FACTOR PRESERVING PRIVACY AUTHENTICATION SYSTEM}

Biometric Authentication (BA) of every grid member can be used to enhance the security of the grid environment. The biometric authentication involves using a smart card which holds the data of what the user is i.e., the biometric data such as finger print, iris, etc, what the user knows i.e., the password (PW) and the data that the user has i.e., Identifier (ID). The 4F2PB method has four phases such as Initialization phase, Registration Phase, Login phase and a password change phase. The first three phases are based on the authentication scheme described in [16] with some additional features. The added features are the dynamic User ID (CID), the dynamic server ID (SID) and the position location feature for the user $\left(\operatorname{pos}_{\mathrm{i}}\right)$ and the server $\left(\operatorname{pos}_{\mathrm{s}}\right)$. The server supports symmetric and asymmetric encryption and decryption. The notations used in this paper are given in Table 1.
Table 1. Notations used in this Paper

\begin{tabular}{|c|l|}
\hline $\mathrm{S}_{\mathrm{i}}$ & Server \\
\hline $\mathrm{U}_{\mathrm{i}}$ & User \\
\hline $\mathrm{S}_{\mathrm{ID}}$ & Identity of Server $\mathrm{S}_{\mathrm{i}}$ \\
\hline $\mathrm{ID}_{\mathrm{i}}$ & Identity of User $\mathrm{U}_{\mathrm{i}}$ \\
\hline $\mathrm{B}_{\mathrm{i}}$ & Biometric data of $\mathrm{U}_{\mathrm{i}}$ \\
\hline$(\mathrm{PW}$ & Password of $\mathrm{U}_{\mathrm{i}}$ \\
\hline $\mathrm{h}()$. & One-way hash function \\
\hline $\mathrm{X}$ & The master secret key \\
\hline$\left(\mathrm{p}_{\mathrm{k}}, \mathrm{s}_{\mathrm{k}}\right)$ & Public-private key pair \\
\hline$\oplus$ & The Exclusive-OR Operation \\
\hline$\|$ & Message Concatenation \\
\hline $\mathrm{r}$ & Random Number generated by $\mathrm{U}_{\mathrm{i}}$ \\
\hline $\mathrm{n}_{\mathrm{u}}$ & Nonce value generated by $\mathrm{U}_{\mathrm{i}}$ \\
\hline $\mathrm{n}_{\mathrm{S}}$ & Nonce value generated by the server $\mathrm{S}_{\mathrm{i}}$ \\
\hline $\mathrm{pos}_{\mathrm{i}}$ & Position of the user $\mathrm{U}_{\mathrm{i}}$ \\
\hline $\mathrm{pos}_{\mathrm{S}}$ & Position of the server $\mathrm{S}_{\mathrm{i}}$ \\
\hline
\end{tabular}

\subsection{Initialization Phase}

During the initialization phase, the server generates a publicprivate key pair. The server also generates a secret key for symmetric encryption and decryption. The server keeps the private key and the secret key secure. 
The following are the series of steps done in the Initialization phase:

a. Server generates public-private key pair $\left(\mathrm{p}_{\mathrm{k}}, \mathrm{s}_{\mathrm{k}}\right)$ for asymmetric encryption/decryption.

b. Server generates a secret key $\mathbf{x}$ for symmetric encryption/decryption.

c. Both $\left(\mathrm{X}, \mathrm{S}_{\mathrm{k}}\right)$ are kept secure in the server.

\subsection{Registration Phase}

During the registration phase, the user tries to register for a grid membership within a Virtual Organization (VO). During the membership registration, the user is given a particular Identifier $\left(\mathrm{ID}_{\mathrm{i}}\right)$. The user registers his/her biometric data $\left(\mathrm{B}_{\mathrm{i}}\right)$ which maybe a fingerprint or an iris template. The user also selects a random number $\mathrm{r}$ and a password $\left(\mathrm{PW}_{\mathrm{i}}\right)$.

The operations done at the user side are:

a. The user records his/her user Identifier $\left(\mathrm{ID}_{\mathrm{i}}\right)$

b. The user records the biometric template $\left(\mathrm{B}_{\mathrm{i}}\right)$

c. The user selects a random string $\mathrm{r}$ and password $\left(\mathrm{PW}_{\mathrm{i}}\right)$

a. The user computes $S B_{i}=\delta\left(B_{i}\right)=h\left(r \oplus B_{i}\right)$. The value of $S_{B}$ is sent to the server securely along with the one-way hash function $\mathrm{h}($.) of the Password and the ID of the user. The server receives $\left(\mathrm{ID}_{\mathrm{i}}, \mathrm{h}\left(\mathrm{PW}_{\mathrm{i}}\right), \mathrm{SB}_{\mathrm{i}}\right)$ through a secure channel. By using the values sent by the user, the server computes, $\mathrm{y}_{\mathrm{i}}$ such that,

$\mathrm{y}_{\mathrm{i}}=\mathrm{E}_{\mathrm{x}}\left(\mathrm{ID}_{\mathrm{i}}\left\|\mathrm{h}\left(\mathrm{PW}_{\mathrm{i}}\right)\right\| \mathrm{SB}_{\mathrm{i}}\right)$

where, $\mathrm{E}_{\mathrm{X}}($.$) represents the symmetric encryption operation$ using the secret key $\mathrm{x}$.

The operations continued in the server side are:

a. Server computes $\mathrm{K}=\mathrm{h}\left(\mathrm{ID}_{\mathrm{i}} \| \mathrm{x}\right)$

b. Server stores $\left(K, y_{i}, h(),. p_{k}\right)$ in the smart card.

c. Server sends smart card to the user securely.

Once the user receives the smart card, a few entries are to be stored in it along with the data already available in the smart card i.e., $\mathrm{y}_{\mathrm{i}}$.

The following operations are done to confirm the registration:

a. The user enters the biometric data which can be an iris data /fingerprint $\mathrm{B}_{\mathrm{i}}$

b. The user encrypts the random number $r$ with $B_{i}$ such that $\mathrm{E}_{\mathrm{B}_{\mathrm{i}}}(\mathrm{r})$ is obtained.

c. $\mathrm{E}_{\mathrm{B}_{\mathrm{i}}}(\mathrm{r})$ - stored in the smart card.

d. $\quad \mathrm{SB}_{\mathrm{i}}=\delta\left(\mathrm{B}_{\mathrm{i}}\right)=\mathrm{h}\left(\mathrm{r} \oplus \mathrm{B}_{\mathrm{i}}\right)$ is stored in the smart card.

\subsection{Login Phase}

A user $\mathrm{U}_{\mathrm{i}}$ is allowed to enter the grid environment using his/her smart card. The user enters his/her Password (PW)' and does a biometric scan denoted by $\mathrm{B}_{\mathrm{i}}^{*}$. The user's smart card retrieves the random value " $r$ " from $E_{B_{i}}(r)$ by using the biometric data $B_{i}^{\prime}$ entered by the user $U_{i}$. The smart card computes $\mathrm{SB}_{\mathrm{i}}{ }^{*}=\delta\left(\mathrm{SB}_{\mathrm{i}}^{\prime}\right)=\mathrm{h}\left(\mathrm{r} \oplus \mathrm{B}_{\mathrm{i}}^{\prime}\right)$. This value is compared with the already stored value of $\mathrm{SB}_{\mathrm{i}}=$ $\delta\left(B_{i}\right)=h\left(r \oplus B_{i}\right)$ to confirm if the user is the same.
Then the smart card generates a nonce value " $\mathrm{n}_{\mathrm{u}}$ " and computes $\mathrm{M}=\left(\mathrm{K} \oplus \mathrm{n}_{\mathrm{u}}\right)$. Then $\mathrm{CID}_{\mathrm{i}}$ is calculated such that, $\mathrm{CID}_{\mathrm{i}}=\mathrm{h}\left(\mathrm{ID}_{\mathrm{i}} \| \mathrm{n}_{\mathrm{u}}\right)$

Then value of $C_{0}$ is computed such that,

$$
\mathrm{C}_{0}=\mathrm{E}_{\mathrm{p}_{\mathrm{k}}}\left(\mathrm{M}\left\|\mathrm{CID}_{\mathrm{i}}\right\| \mathrm{y}_{\mathrm{i}}\|\mathrm{u}\| \operatorname{pos}_{\mathrm{i}}\right)
$$

Where $\mathrm{E}_{\mathrm{p}_{\mathrm{k}}}($.$) denotes the encryption function using the$ server's public key. " $\mathrm{u}$ " is the random value selected by the user during login time. pos $_{i}$ - denotes the position at which the user is during this phase.

To ensure the liveliness of the user, a nonce value is added in the value of $\mathrm{C}_{0}$ along with the already existing random values to add more security. $\mathrm{C}_{0}$ is sent to the server.

\subsection{Mutual Authentication Phase}

Once $\mathrm{C}_{0}$ is received by the server, the server does the following operations,

a. Server decrypts $\mathrm{C}_{0}$ using its private key $\mathrm{s}_{\mathrm{k}}$

b. $\quad$ Server computes " $n_{u}^{*}$ " such that $n_{u}^{*}=M \oplus K$ where $\mathrm{K}=\mathrm{h}\left(\mathrm{ID}_{\mathrm{i}} \| \mathrm{x}\right)$

c. The validity of the user is checked by using the Identifier $\mathrm{ID}_{\mathrm{i}}$ to the one received by the server. By using the value of $\mathrm{n}_{\mathrm{u}}^{*}$ the value of $\mathrm{CID}_{\mathrm{i}}^{*}$ is calculated.

d. Then the value of $\operatorname{CID}_{\mathrm{i}}^{*}$ is compared with the value of $\mathrm{CID}_{\mathrm{i}}$ to check if $\mathrm{CID}_{\mathrm{i}}=\mathrm{CID}_{\mathrm{i}}^{*}$.

e. Also the value of $\mathrm{ID}_{\mathrm{i}}$ can be verified with the ID stored in the ID table for the users at the server end. A comparison of ID's is done to make sure that verification is done correctly even when the Server ID table is corrupted.

f. The remaining terms of $\mathrm{C}_{0}$ i.e., $\left(\mathrm{h}\left(\mathrm{PW}_{\mathrm{i}}\right) \| \mathrm{SB}_{\mathrm{i}}\right)$ is retained for future reference.

Server computes a values of $\mathrm{C}_{1}$ such that

$\mathrm{C}_{1}=\mathrm{E}_{\mathrm{u}}\left(\mathrm{N}\|\mathrm{SED}\| \mathrm{S}_{\mathrm{ID}}\|\mathrm{v}\| \operatorname{pos}_{\mathrm{s}}\right)$

Where $S_{I D}=$ Server's identity and $v$ is the random number chosen by the server and $\mathbf{u}$ is the random number selected by the user and sent in $\mathrm{C}_{0}$. The server generates a nonce value " $\mathrm{n}_{\mathrm{s}}$ " and computes $\mathrm{N}=\left(\mathrm{K} \oplus \mathrm{n}_{\mathrm{s}}\right)$. pos ponotes $_{\mathrm{s}}$ the position of where the server is during the authentication session and Server ID SED $=h\left(S_{\text {ID }} \| n_{s}\right)$. This is done to make sure that the data was not tampered during transmission. Server sends $C_{1}$ to the user $U_{i}$.

In the User Side, the following operations are done,

a. The smart card decrypts $C_{1}$ using the random value of u.

b. The value of $S_{\text {ID }}$ is checked for valid server ID. The smart card computes $\mathrm{SED}^{*}=\mathrm{h}\left(\mathrm{S}_{\mathrm{ID}} \| \mathrm{n}_{\mathrm{s}}^{*}\right)$ using its nonce value $n_{\mathrm{s}}^{*}$. Smart Card computes " $\mathrm{n}_{\mathrm{s}}^{*}$ " such that $\mathrm{n}_{\mathrm{s}}^{*}=\mathrm{N} \oplus \mathrm{K}$ where $\mathrm{K}=\mathrm{h}\left(\mathrm{ID}_{\mathrm{i}} \| \mathrm{x}\right)$.

c. Then $\mathrm{SED}^{*}$ is calculated by using the value of the generated $n_{s}^{*}$ and $I_{i}$, i.e., $S E D^{*}=h\left(S_{I D} \| n_{s}^{*}\right)$. If 
$\mathrm{SED}^{*}=\mathrm{SED}$, then the server is valid and the data has not been tampered with.

d. The position of the server is stored by the user for further use.

The smart card calculates the following value

$$
\mathrm{C}_{2}=\mathrm{E}_{\mathrm{v}}\left(\mathrm{h}\left(\mathrm{PW}_{\mathrm{i}}\right)^{\prime}\left\|\mathrm{SB}_{\mathrm{i}}{ }^{\prime}\right\| \operatorname{pos}_{\mathrm{i}}\right)
$$

Where $\operatorname{pos}_{\mathrm{i}}$ denotes the position at which the user is during this instant.

The server decrypts $C_{2}$ using $\mathrm{v}$ and calculates the value of $y_{i}^{*}$ from the values sent in $C_{2}$. If $y_{i}^{*}=y_{i}$, the server matches the values of the password and the biometric template to confirm the authenticity of the user. Finally, the server checks the position of the user.

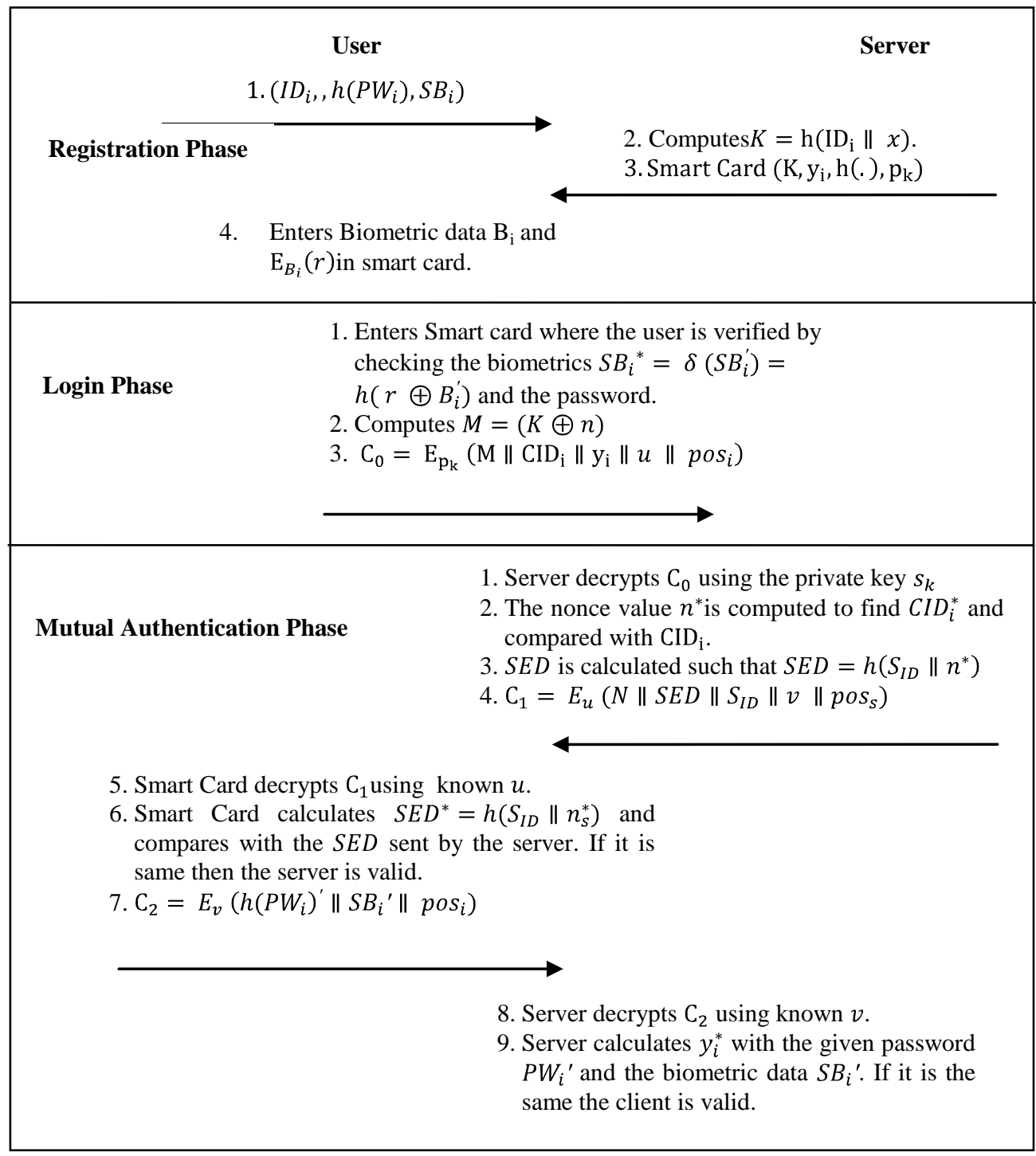

Figure 2. Proposed 4F2PB Authentication Scheme

The position of the user cannot change drastically between $\mathrm{C}_{0}$ and $\mathrm{C}_{2}$ thereby indicating to the server that there is no presence of an attacker. If an attacker is to attack, he/she has to position themselves in the in the correct position and the nonce all in once which makes the attack much difficult. The value of $\mathrm{SB}_{\mathrm{i}}^{*}$ in $\mathrm{C}_{2}$ is compared with $\mathrm{SB}_{\mathrm{i}}$ of $\mathrm{y}_{\mathrm{i}}$. If the value match is within a threshold range then the user is confirmed valid. The flow of the proposed scheme $4 \mathrm{~F} 2 \mathrm{~PB}$ is shown in fig. 2. The main phases three phases are considered for computing the cost since they will be used repeatedly. Once all the steps in fig. 2 have been completed successfully, it is clear that mutual authentication of both the user and the server is done for login of the user. The server secret number $\mathrm{V}$ can be used as a session key material and $h(v)$ can be used as a session key which is shared with the server. The triple DES along with any other light-weight 
encryption algorithm can be use for the purpose of encryption.

\subsection{Password Change Phase}

The user $\mathrm{U}_{\mathrm{i}}$ is authenticated by using the Password ( $\mathrm{PW}$ ) used initially for login process. Once authenticated, the user is prompted to enter the new password. Once the new password (PW") is entered, the $\mathrm{y}_{\mathrm{i}}=\mathrm{E}_{\mathrm{x}}\left(\mathrm{ID}_{\mathrm{i}} \|\right.$ $\left.h\left(P W_{i}\right) \| S_{i}\right)$ value of $h\left(P W_{i}\right)$ is replaced with $\mathrm{h}\left(\mathrm{PW}_{\mathrm{i}}{ }^{\prime \prime}\right)$. Thereby the user is allowed to further login by using the new password. The next section gives a brief discussion on the security analysis of the 4F2PB authentication scheme.

\section{SECURITY ANALYSIS OF 4F2PB AUTHENTICATION METHOD}

In this section, the security and performance analysis of the 4F2PB authentication scheme are presented. The attacks which are withstood by the proposed scheme of authentication are explained.

\subsection{ID - Theft Attack}

As in equation $\mathrm{C}_{0}=\mathrm{E}_{\mathrm{p}_{\mathrm{k}}}\left(\mathrm{M}\left\|\mathrm{CID}_{\mathrm{i}}\right\| \mathrm{y}_{\mathrm{i}}\|\mathrm{u}\|\right.$ $\operatorname{pos}_{\mathrm{i}}$ ), a dynamic user ID named as $\mathrm{CID}_{\mathrm{i}}$ is created by the smart card based on the nonce value $\mathrm{n}_{\mathrm{u}}$ instead of using the user's own ID. This helps to withstand the ID-theft attack and also preserves the privacy of the user.

\subsection{Clock Synchronization and Replay Attack Problem}

In [19], the problem in timestamp based authentication is given as replay attack due to the transmission delays in an unpredictable network. Even though the networks are fast the speed may vary based on the geographical and political distribution. To avoid using of timestamps, a nonce value $\mathrm{n}_{\mathrm{u}}$ is used each time the user sends his/her data and a nonce value $n_{s}$ is used by the server to declare the server's validity. Since a nonce value such as $\mathrm{n}_{\mathrm{u}}$ and $\mathrm{n}_{\mathrm{s}}$ in equations $\quad \mathrm{C}_{0}=\mathrm{E}_{\mathrm{p}_{\mathrm{k}}}\left(\mathrm{M}\left\|\mathrm{CID}_{\mathrm{i}}\right\| \mathrm{y}_{\mathrm{i}}\|\mathrm{u}\| \operatorname{pos}_{\mathrm{i}}\right)$ where $\mathrm{M}=\left(\mathrm{K} \oplus \mathrm{n}_{\mathrm{u}}\right)$ and $\mathrm{C}_{1}=\mathrm{E}_{\mathrm{u}}(\mathrm{N}\|\mathrm{SED}\|$ $\left.\mathrm{S}_{\mathrm{ID}}\|\mathrm{v}\| \mathrm{pos}_{\mathrm{s}}\right)$ where $\mathrm{N}=\left(\mathrm{K} \oplus \mathrm{n}_{\mathrm{s}}\right)$ can be used only once, and not repeated, the user/server can be safeguard themselves from replay attacks.

\subsection{Modification Attack}

Each authentication message in from equation (1), (2), (3) and (4) include a one-way hash function along with an encryption algorithm. The hash value in each equation requires a nonce value or a random value. Even if the attacker gets hold of each of these equations the decryption part and breaking the hash function is not possible. If the attacker has the value of $h\left(\mathrm{PW}_{\mathrm{i}}\right)$, to find the password, the attacker needs find an equivalent of the hash function by trying each password. This attack is difficult to occur because the attacker has to first break into the encrypted data $\mathrm{C}_{0}=\mathrm{E}_{\mathrm{p}_{\mathrm{k}}}\left(\mathrm{M}\left\|\mathrm{CID}_{\mathrm{i}}\right\| \mathrm{y}_{\mathrm{i}}\|\mathrm{u}\| \operatorname{pos}_{\mathrm{i}}\right)$. The attacker then needs to send the correct dynamic ID using the nonce and the position data $\operatorname{pos}_{\mathrm{i}}$. For an attacker to get all the values correct is impossible which makes modification attack difficult. Without knowing the actual data of these two values, the original data cannot be modified. Modification of the equations will be noted by the legitimate user and server and since all the messages are linked, it makes modification attack harder.

\subsection{Mutual Authentication}

At the end of the mutual authentication phase, both the server and the client authenticate each other thereby establishing mutual authentication. During each phase of the equations $\mathrm{C}_{0}, \mathrm{C}_{1}$ and $\mathrm{C}_{3}$, the user and server check the validity of each other using the values of CID, SED, M, N and the position data's. If the server has any doubt in the validity of the user, the message $C_{2}$ can be asked to be resent and the position can be checked.

\subsection{Man-in-the-middle Attack}

An attacker A who tries to do a man-in-the middle attack needs to know the decryption keys such as $u, v$ and $r$ in each message signal else its message will be discarded by the server or the client. The position data pos which is considered as the fourth factor is to be within a threshold for the final authentication to hold valid.

\subsection{Security of the Stored Data on the Smart Card}

The smart card holds the value of $\left(\operatorname{ID}_{\mathrm{i}}, \mathrm{y}_{\mathrm{i}}, \mathrm{h}(),. \mathrm{p}_{\mathrm{k}}\right)$ where, $y_{i}=E_{x}\left(I_{i}\left\|h\left(P W_{i}\right)\right\| S B_{i}\right)$. If the smart card is compromised, the data it provides is not easily accessible to the attacker. Without knowing the matching password and the ID of the user, the attacker cannot move further along the authentication phase. Knowing the public key of the server complicates matters since the attacker has to find the encryption algorithm and a matching value of $\mathrm{C}_{0}$ to send to the server. Furthermore, the hash function has to be broken in order to get the secret data. The biometric data is stored in the open for anyone to copy it. It is stored in the form of a template combined with a random string which needs to be found to get the data. Thus the data stored in the smart card is secure.

\section{PERFORMANCE FUNCTIONALITY ANALYSIS OF THE 4F2PB AUTHENTICATION SCHEME}

In this section, the performance and functionality of the $4 \mathrm{~F} 2 \mathrm{~PB}$ authentication scheme is analysed and comparison has been made with the $\mathrm{X}$. $\mathrm{Li}$ et al. scheme and $\mathrm{Li}$ and Hwang scheme. Where, $\mathrm{T}_{\mathrm{h}}$ denotes the time complexity of the one-way hash function and $\mathrm{T}_{\mathrm{EN}}$ is the time taken to complete one full Encryption algorithm. In [20], X. Li et al. have provided a comparison of their biometric remote authentication system and $\mathrm{Li}$ and Hwang scheme [21] of authentication. The 4F2PB authentication scheme proposed in this paper is compared with the $\mathrm{X}$. $\mathrm{Li}$ et al. scheme and $\mathrm{Li}$ and Hwang scheme as shown in Table 2. 
Table 2. Performance Comparison of the 4F2PB Scheme with Other Biometric Schemes

\begin{tabular}{|l|c|c|c|}
\hline \multicolumn{1}{|c|}{ Phase } & $\begin{array}{c}\text { 4F2PB } \\
\text { Scheme }\end{array}$ & $\begin{array}{c}\text { X. Li et } \\
\text { al. } \\
\text { Scheme } \\
{[\mathbf{1 9}]}\end{array}$ & $\begin{array}{c}\text { Li and } \\
\text { Hwang } \\
\text { Scheme } \\
{[\mathbf{2 0}]}\end{array}$ \\
\hline $\begin{array}{l}\text { Registration } \\
\text { Phase }\end{array}$ & $1 \mathrm{~T}_{\mathrm{EN}}+2 \mathrm{~T}_{\mathrm{h}}$ & $4 \mathrm{~T}_{\mathrm{h}}$ & $3 \mathrm{~T}_{\mathrm{h}}$ \\
\hline Login Phase & $1 \mathrm{~T}_{\mathrm{EN}}+1 \mathrm{~T}_{\mathrm{h}}$ & $4 \mathrm{~T}_{\mathrm{h}}$ & $2 \mathrm{~T}_{\mathrm{h}}$ \\
\hline $\begin{array}{l}\text { Mutual } \\
\text { Authentication } \\
\text { Phase }\end{array}$ & $\begin{array}{c}3 \mathrm{~T}_{\mathrm{EN}} \\
+2 \mathrm{~T}_{\mathrm{h}}\end{array}$ & $7 \mathrm{~T}_{\mathrm{h}}$ & $5 \mathrm{~T}_{\mathrm{h}}$ \\
\hline Total & $\begin{array}{c}5 \mathrm{~T}_{\mathrm{EN}} \\
+5 \mathrm{~T}_{\mathrm{h}}\end{array}$ & $15 \mathrm{~T}_{\mathrm{h}}$ & $10 \mathrm{~T}_{\mathrm{h}}$ \\
\hline
\end{tabular}

The Table 2 has been generated based on the time taken for each phase. The Registration phase involves the time taken for two has function and one encryption algorithm; the login phase involves the time taken for one hash function and one encryption algorithm and the Mutual Authentication phase has a total time taken as a combination of three encryption operations and two hash functions. The overall time taken to complete the 4F2PB scheme of authentication is much less when compared to the existing biometric authentication systems. This is an added advantage of the 4F2PB authentication scheme.

The Table 3 gives the overall security comparison for $\mathrm{X}$. Li et al. scheme and Li and Hwang scheme with our proposed 4F2PB algorithm. The four factor algorithm takes a much lesser time with more security when compared with X. Li et al. scheme and $\mathrm{Li}$ and Hwang scheme. Since a dynamic ID is used for the user with a combination of a nonce value, the 4F2PB algorithm resists ID-theft attack whereas X. Li et al. scheme and Li and Hwang scheme use the ID of the user and hence are vulnerable to such attacks.

Table 3. Security Comparison of the 4F2PB Scheme with other Biometric Schemes

\begin{tabular}{|l|c|c|c|}
\hline \multicolumn{1}{|c|}{ Factors } & 4F2PB scheme & $\begin{array}{c}\text { X. Li et al. } \\
\text { Scheme [19] }\end{array}$ & $\begin{array}{c}\text { Li and Hwang } \\
\text { Scheme [20] }\end{array}$ \\
\hline Computational Cost & Low $\left(5 \mathrm{~T}_{\mathrm{EN}}+5 \mathrm{~T}_{\mathrm{h}}\right)$ & Low $\left(15 \mathrm{~T}_{\mathrm{h}}\right)$ & ${\text { Low }\left(10 \mathrm{~T}_{\mathrm{h}}\right)}$ \\
\hline Mutual Authentication & Yes & Yes & No \\
\hline Resistance to replay attack & Yes & Yes & Yes \\
\hline Resistance to modification & Yes & Yes & No \\
\hline Resistance to man-in-the-middle attacks & Yes & Yes & 3 \\
\hline Factors in Authentication & 4 & 3 & No \\
\hline Matching Biometric data in remote server & Yes & No & No \\
\hline Resist ID theft & Yes & No &
\end{tabular}

The biometric matching is not done mostly in the smart card in $4 \mathrm{~F} 2 \mathrm{~PB}$ but rather in the remote server without losing the privacy of the biometric data.

The Exclusive OR operation requires less computational time thereby cost when compared to the hash function and encryption operations and hence the cost of it is neglected. Any light-weight public-key cryptosystem can be used for the encryption and decryption process.

\section{AUTHORIZATION SCHEME USING BIOMETRIC DATA}

Authorization involves primarily the process of providing the access control to the users for the resources. Access Control in our architecture is based on Role Based Access Control (RBAC) [22]. For providing RBAC, some sets of policies are to be formulated for the Grid Environment and the corresponding virtual organizations. The policies are formulated based on the combination of the Domain policy to which the user is a part, the VO policy of which the user is a member and the user's own requirements. RBAC is implemented using the XACML/SAML for appropriate access details.

The access control is implemented by the collaboration between the policy decision points (PDPs) and policy enforcement points (PEPs). PDPs perform authorization decisions; whereas PEPs carry out the access decisions made by the PDPs. In this security architecture, PDP's are the Security Manager and PEP's are the Security Clients. The Authentication Policy (AuP) and Authorization Policy (AuZP) are formulated in the Virtual Organization Policy set (VP). The final effect of the policy is either a Permit a Deny. Fig. 3 gives the combined structure Authentication and Access Control mechanism for which can be added for this architecture.

The SC and the SM communicate through a secure channel. The SM decides on the access decisions based on the policy set of the VO and the RBAC details of the user. The user's previous history along with the user's present requirements decides the access criteria for the user. The details are stored for future use. This further enhances providing the access to the user. 


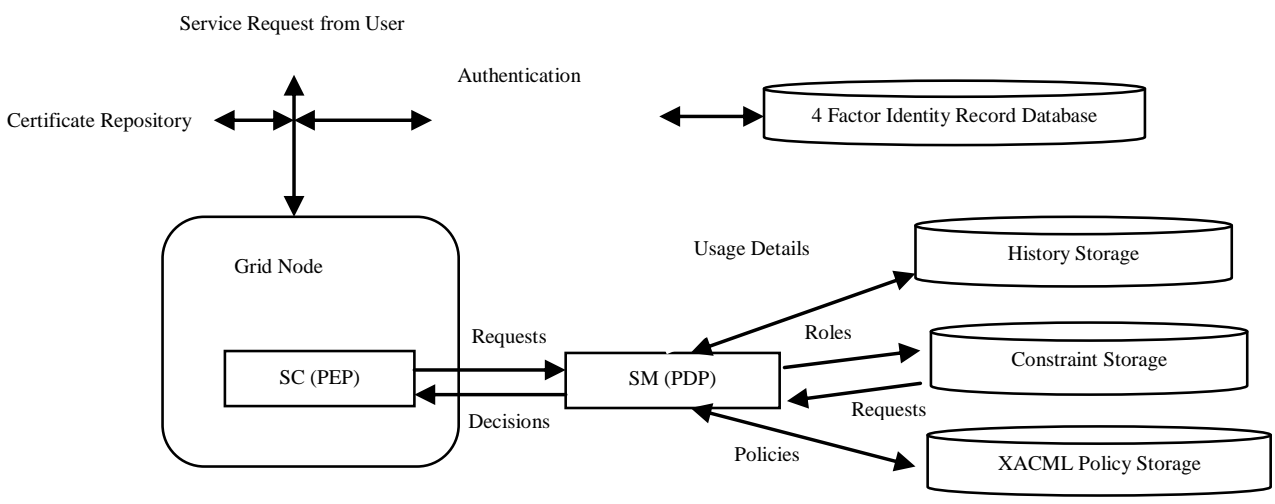

Figure 3. Authorization Scheme Combined with the Authentication Scheme

In the Fig.3, the PEP and the PDP are the Security Client and the Security Manager of proposed basic architecture. As in Fig.3, the user gets authenticated to enter into the grid environment. After authentication to access any resource or service, the user's viability is finalized with the roles provided to the user, his /her usage history and the policyset of the service. The biometric data of the user is linked with his/her roles for access of the required resource [23]. The resource access is based on the policy set of the resource provider and the rights provided to the user.

\section{CONCLUSION AND FUTURE WORK}

The proposed Four-Factor based Privacy Preserving Biometric authentication scheme has provided an enhanced security with an optimal overall time taken for the operation. The scheme provides a four factor authentication with better security prospects for any user in the grid network. By increasing the security during the authentication phase itself we can try to minimize any other malicious insider attacks and also reduce external attacks. The biometric data used for authentication can also be used in the consecutive authorization process thereby lessening the database space utilized by reusing the data used in authentication. A further study in the analysis of the biometric authorization is to be done to check for its viability.

\section{REFERENCES}

[1] Ian Foster, "What is the Grid? A Three Point Checklist", 2002.

[2] Foster, C. Kesselman, S. Tuecke, "The anatomy of the grid: enabling scalable virtual organizations", Int. J. High Performance Computing, 2001.

[3] Quan Zhou, Geng Yang, Jiangang Shen, Chunming Rong, "A Scalable Architecture for Grid", Sixth International Conference on Parallel and Distributed Computing, Applications and Technologies, 2005.

[4] Bendahmane, M. Essaaidi, A. El Moussaoui, A. Younes, "Grid Computing Security Mechanisms: Stateof-The-Art", International Conference on Multimedia Computing and systems ICMS ’09, pp535-540, 2009.

[5] The Globus Security Team, Globus Toolkit Version 4 Grid Security Infrastructure: A Standards Perspective, Version 4 updated September 12, 2005, http://www.globus.org/toolkit/docs/4.0/security/GT4GSI-Overview.pdf
[6] Lamport. L., "Password Authentication with insecure Communication", ACM Communications 24(11), 770$772,1981$.

[7] Hwang.T, Chen.Y, Laih. C. S, "Non-Interactive password authentication without password tables", IEEE Conference on Computer and Communication Systems, pp. 429-431.

[8] J.K.Lee, S.R.Ryu and K.Y.Yoo, "Fingerprint-based remote user authentication scheme using smart cards", Electron. Lett., vol.38, no.12, pp.554-555, 2002.

[9] C.C.Chang and I.C.Lin, "Remarks on fingerprint-based remote user authentication scheme using smart cards", ACM SIGOPS operating System Rev., vol.38,no.4, pp. 91-96, 2004.

[10] C.H.Lin and Y.Y.Lai, "A flexible biometrics remote user authentication scheme", Computer Standards Interfaces, vol.27, no.1, pp.19-23, 2004.

[11] U. Uludag, S. Pankanti, S. Prabhakar, and A. K. Jain, "Biometric cryptosystems: Issues and challenges," Proc. IEEE, Special Issue on Multimedia Security for Digital Rights Management, vol. 92, no. 6, pp. 948960, Jun. 2004.

[12] Y. Dodis, R. Ostrovsky, L. Reyzin, and A. Smith, "Fuzzy extractors: How to generate strong keys from biometrics and other noisy data", in Eurocrypt 2004, pp. 523-540.

[13] A. Juels and M. Wattenberg, "A fuzzy commitment scheme", in Proc. ACMConf. Computer and Communications Security, 1999, pp. 28-36.

[14] Y. Sutcu, Q. Li, and N. Memon, "Protecting biometric templates with sketch: Theory and practice", IEEE Trans. Inf. Forensics Security, vol. 2, no. 3, pp. 503512, Sep. 2007.

[15] C.M. Chen and W.C. Ku, "Stolen-verifier attack on two new strong-password authentication protocol", IEICE transactions on Communications, E85-B (11), 20002, pp. 2519-2521.

[16] Chun-I Fan and Yi-Hui Lin, "Provably secure remote Truly Three-Factor Authentication Scheme with Privacy Protection on Biometrics", IEEE Transactions on information Forensic and Security, vol. 4, No.4, December 2009. 
[17] Dustin D. Trammell, "Four-factor Authentication", http://blog.dustintrammell.com/2008/11/21/four-factorauthentication

[18] The Open Grid Services Architecture, Version 1.5, http://www.ogf.org/documents/GFD.80.pdf

[19] Gong. L, "Security risk of depending on synchronized clocks", ACM Operating System Review", ACM Operating System Review. 26(1), pp. 49-53.

[20] Xiong Li, Jian-Wei Niu, Jian Ma, Wen-Dong Wang, Cheng-Lian Liu, "Cryptanalysis and improvement of a biometrics-based remote user authentication scheme using smart cards", Journal of Network and Computer Applications 34 (2011) pp.73-79.
[21] Li C-T, Hwang M-S,” An Efficient biometrics-based remote user authentication scheme using smart cards", Journal of Network and Computer Applications, 2010, 33(1) pp. 1-5.

[22] Ferraiolo D F, Sandhu R, Gavrila S, "Proposed NIST standard for role-based access control. ACM Transactions on Information and System Security", 2001, 4(3): $224-274$.

[23] Bechara Al Bouna, Richard Chbeir, Stefania Marrara, "Enforcing role based access control model with multimedia signatures", Journal of Systems Architecture 55 (2009) 264-274. 